\title{
Antibodies against PCSK9-a new era of therapy
}

\section{T} he 2012 AHA Scientific Sessions saw considerable interest in the use of monoclonal antibodies against the serine protease PCSK9 (proprotein convertase subtilisin/kexin type 9) in patients with hypercholesterolaemia. Data from at least five phase II trials of patients with various indications for treatment were presented and simultaneously published. Two antibodies were tested-AMG 145 (Amgen, Thousand Oaks, CA, USA) in four of the studies, and SAR236553/REGN727 (Regeneron Pharmaceuticals, Inc., Tarrytown, NY, USA; and Sanofi, Paris, France) in one study.

Despite the success of statin therapy, some patients do not achieve target lipid levels, particularly individuals with a very high LDL-cholesterol (LDL-C) level and those intolerant to statins. Additional treatments to reduce residually high lipid levels are, therefore, required. PCSK9 binds to the LDL receptor preventing the clearance of LDL-C from the blood. Antibody inhibition of PCSK9 has become a novel therapeutic strategy for reducing LDL-C concentration.

In the RUTHERFORD trial, 168 statintreated patients with heterozygous familial hypercholesterolaemia were randomly assigned to receive AMG 145 at $350 \mathrm{mg}$ or $420 \mathrm{mg}$, or a placebo once every 4 weeks for 12 weeks. AMG 145 dramatically reduced the LDL-C level-by $43 \%$ for $350 \mathrm{mg}$ and by $55 \%$ for $420 \mathrm{mg}$ - compared with placebo. Overall, $70 \%$ and $80 \%$ of patients receiving low-dose and high-dose AMG 145, respectively, achieved the target LDL-C level of $<100 \mathrm{mg} / \mathrm{dl}$ ( $44 \%$ and $65 \%$ achieved $<70 \mathrm{mg} / \mathrm{dl}$ ). The triglyceride level was also reduced, and the HDL-cholesterol level modestly, but significantly, increased with AMG 145. No substantial safety concerns were raised.

The GAUSS trial involved 160 patients with hypercholesterolaemia who were statin intolerant owing to myalgia. Five treatment groups were defined-AMG 145 alone ( $280 \mathrm{mg}$, $350 \mathrm{mg}$, or $420 \mathrm{mg}$ ),

AMG $145420 \mathrm{mg}$ plus ezetimibe $10 \mathrm{mg}$, or ezetimibe $10 \mathrm{mg}$ plus a placebo. Treatment was given every 4 weeks for 12 weeks. A dose-dependent reduction in LDL-C level occurred with AMG 145 alone, ranging from $41 \%$ with $280 \mathrm{mg}$ to $51 \%$ with $420 \mathrm{mg}$. The reduction was even greater $(63 \%)$ with the antibody plus ezetimibe. Levels of other non-HDL-cholesterols were reduced, and the HDL-cholesterol level increased. Seven patients taking AMG 145 alone experienced myalgia, but this effect was not dose-related.

In the LAPLACE-TIMI 57 trial, various doses of AMG 145 were compared with placebo in 631 patients with hypercholesterolaemia despite receiving statin therapy. Patients were randomly assigned to receive $70 \mathrm{mg}, 105 \mathrm{mg}$, or $140 \mathrm{mg}$ of AMG 145, or a placebo every 2 weeks, or $280 \mathrm{mg}, 350 \mathrm{mg}$, or $420 \mathrm{mg}$ of AMG 145, or a placebo every 4 weeks for 12 weeks. Again, AMG 145 dosedependently reduced the level of LDL-C, with the greatest reduction $(66.1 \%)$ achieved with $140 \mathrm{mg}$ every 2 weeks. Overall, doses every 2 weeks produced greater reductions in LDL-C than doses every 4 weeks. The target LDL-C level of $70 \mathrm{mg} / \mathrm{dl}$ was reached in $94 \%$ of patients who received AMG 145140 mg every 2 weeks. No difference in adverse events between any of the doses, or between AMG 145 and placebo, was recorded.

The MENDEL trial investigators assessed the use of AMG 145 among 406 statin-naive patients with hypercholesterolaemia. The same dosing schedules of AMG 145 as in the LAPLACE-TIMI 57 trial were used. In addition, one group was assigned to receive oral ezetimibe $10 \mathrm{mg}$ per day. Again, the greatest reduction in LDL-C level (50.9\%) was achieved with AMG 145140 mg every 2 weeks. No differences were reported in LDL-C level reductions at week 12 between the groups receiving AMG $145140 \mathrm{mg}$ every 2 weeks and $420 \mathrm{mg}$ every 4 weeks. AMG 145 was well tolerated.

In a study by Roth and colleagues, another antibody against PCSK9, SAR236553/REGN727, was tested in 92 statin-treated patients with residual

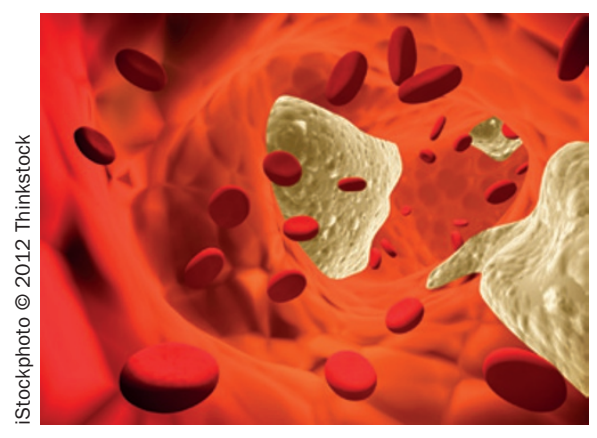

hypercholesterolaemia. Participants were assigned to either atorvastatin $10 \mathrm{mg}$ per day or $80 \mathrm{mg}$ per day plus the antibody every 2 weeks, or to atorvastatin $80 \mathrm{mg}$ per day plus a placebo for 8 weeks. SAR236553/ REGN727 plus $10 \mathrm{mg}$ or $80 \mathrm{mg}$ atorvastatin produced a $66 \%$ and $73 \%$ reduction in LDL-C level, respectively. All patients who received the antibody achieved the target LDL-C level of $<100 \mathrm{mg} / \mathrm{dl}$. No significant safety issues were reported. Phase III trials of monoclonal antibodies against PSCK9 are now eagerly awaited.

\section{Alexandra King}

Original articles Raal, F. et al. Low-density lipoprotein
cholesterol-lowering effects of AMG 145, a monoclonal
antibody to proprotein convertase subtilisin/kexin type 9
serine protease in patients with heterozygous familial
hypercholesterolemia: the Reduction of LDL-C with PCSK9
Inhibition in Heterozygous Familial Hypercholesterolemia
Disorder (RUTHERFORD) randomized trial. Circulation
126, 2408-2417 (2012) | Sullivan, D. et al. Effect
of a monoclonal antibody to PCSK9 on low-density
lipoprotein cholesterol levels in statin-intolerant patients:
the GAUSS randomized trial. JAMA doi:10.1001/
jama.2012.25790 | Giugliano, R. P. et al. Efficacy, safety,
and tolerability of a monoclonal antibody to proprotein
convertase subtilisin/kexin type 9 in combination with a
statin in patients with hypercholesterolaemia (LAPLACE-
TIMI 57): a randomised, placebo-controlled,
dose-ranging, phase 2 study. Lancet doi:10.1016/
S0140-6736(12)61770-X | Koren, M. J. et al. Efficacy,
safety, and tolerability of a monoclonal antibody to proprotein
convertase subtilisin/kexin type 9 as monotherapy in patients
with hypercholesterolaemia (MENDEL): a randomised,
double-blind, placebo-controlled, phase 2 study. Lancet
doi:10.1016/S0140-6736(12)61771-1 | Roth, E. M. et al.
Atorvastatin with or without an antibody to PCSK9 in primary
hypercholesterolemia. N. Engl. J. Med. doi:10.1056/
NEJMoa1201832

